\title{
A Novel Media Optimized for Production of Pullulan in Flask Type Fermentation System
}

\author{
Ranjan Singh ${ }^{1 *}$, Rajeeva Gaur ${ }^{2}$, Prabhash K. Pandey ${ }^{3}$, Farrukh Jamal ${ }^{4}$, \\ Laxmi K. Pandey ${ }^{5}$, Sangram Singh ${ }^{4}$, Harish K. Kewat ${ }^{6}$, Soni Tiwari ${ }^{2}$, \\ Pritha Biswas ${ }^{1}$ and Manogya K. Gaur ${ }^{7}$
}

${ }^{I}$ Department of Botany and Microbiology, St. Aloysius College (Autonomous), Jabalpur, M.P., India

${ }^{2}$ Department of Microbiology (DST-FIST Supported, Centre of Excellence, Govt. of U.P.), Dr. R.M.L. Avadh University, Faizabad, U.P., India

${ }^{3}$ Genotoxicity Lab, Division of Toxicology, Central Drug Research Institute, Lucknow, U.P., India

${ }^{4}$ Department of Biochemistry, Dr. R.M.L. Avadh University, Faizabad, U.P., India

${ }^{5}$ Department of Biotechnology, St. Aloysius College (Autonomous), Jabalpur, M.P., India

${ }^{6}$ Regional Office, I.G.N.O.U., Jabalpur, M.P., India

${ }^{7}$ D.G.M. Environment (ETP and Biocompost), Balrampur Distillery, Bhabhanan, Basti, U.P., India

*Corresponding author

\section{Keywords}

Aureobasidium pullulans, Pullulan, Sugarcane juice, Production

Article Info

Accepted:

04 March 2018

Available Online: 10 April 2018

\section{A B S T R A C T}

Aureobasidium pullulans, popularly known as black yeast, is one of the most widespread saprophytic fungus associated with wide range of terrestrial and aquatic habitats. The fungus has widely been employed in production of an economically important polysaccharide Pullulan. Pullulan is a linear glucan made mainly of maltotriose repeating units. This gives the polysaccharide structural flexibility and enhances solubility. This polysaccharide is of great economic importance with increase applications in food, pharmaceutical, agriculture, blood plasma substitute and chemical industry. Production of pullulan was observed in a novel media consisting of Sugarcane juice (SCJ) and distilled water. It was found that high amount of pullulan $(6.4 \pm 0.04 \mathrm{~g} / 100 \mathrm{ml})$ was obtained at $37^{\circ} \mathrm{C}, \mathrm{pH} 5$ at a concentration of $50 \%$ media in $96 \mathrm{~h}$. This is a novel study in which pullulan is grown on media consisting of Sugar Cane Juice (SCJ) and distilled water. Further no synthetic minerals are used in the media for the growth of polysaccharide making it very economical. 


\section{Introduction}

Aureobasidium pullulans (De Bary) Arnaud is cosmopolitan yeast like fungus that occurs in diverse habitats, including the phylloshere of many plants and also on various tropical fruits. A. pullulans is industrially important because of its capacity to produce the polysaccharide Pullulan. Pullulan is a transparent, colorless, tasteless, odorless, tenacious, resistant to oil and grease and unaffected by small thermal variations. It is soluble in cold and hot water and insoluble in organic solvents. It is a linear $\alpha$-D-glucan, made mainly of maltotriose repeating units interconnected by $-1,6$ linkages. The regular alternation of -1, 4 and 1,6 bonds results in two distinctive properties that is structural flexibility and enhanced solubility. This polysaccharide is of great economic importance with increased applications in food, pharmaceutical, agricultural, blood plasma substitute and chemical industries (Gaur, Sing, Gupta and Gaur, 2010; Singh, Gaur, Bansal, Biswas, Pandey et al., 2015).

The cost of Pullulan primarily depends on the raw materials, especially of carbon source, which play a major role in the economics of pullulan production. The sugars such as sucrose, glucose, fructose, maltose, starch support pullulan production by $A$. pullulans (Singh, Gaur, Tiwari and Gaur, 2012; Singh, Gaur, Bansal, Biswas, Pandey et al., 2016; Singh, Gaur, Bansal, Jamal, Pandey et al., 2017). A number of complex carbon sources have been reported for pullulan production, including peat hydrolysate, cornmeal hydrolysates, corn syrup, fuel ethanol fermentation stillage, carob pod, grape skin pulp, olive oil and sucrose, beet molasses, hydrolyzed potato starch, spent grain liquor, jaggery, cashew fruit juice, coconut water and milk, Jerusalem artichoke and on mixture of potato starch hydrolysate and sucrose (Boa and LeDuy, 1984; Boa and LeDuy, 1987;
Leather and Gupta, 1994; Roukas and Biliaderis, 1995; Isralidies, Smith, Harthill, Barnett, Bambalov and Scanlon, 1998; Youseef, Biliaders and Roukas, 1998; Roukas, 1998; Roukas and Liakopoulou, 1999; Barnett, Smith, Scanlon, Israilides, 1999; Roukas, 1999; Vijayendra, Bansal, Prasad and Nand, 2001; Thirumavalan, Manikkandan and Dhaneseskar, 2008; Thirumavalan, Manikkandan and Dhaneseskar, 2009; Xia, $\mathrm{Xu}$, Liu, Xu, Wang and Li, 2017; Chao, Ma, Chang and Xue, 2017).

India is one of the biggest producers of Sugarcane around the world. Sugar Cane Juice (SCJ) contains different sugar and minerals and therefore it acts as a natural growth media for different microorganisms including Aureobasidium pullulans. It contains sugars like sucrose, glucose, and salts like potassium, calcium which is necessary for the growth. In India the juice is available readily and is very cheap. It is consumed as a sweet beverage and added in different food products. Bedside India Sugar Cane is also grown in countries like Brazil, West Indies, Pakistan, U.S.A. and other tropical countries. In the present study pullulan was grown on the media composed from sugar cane juice (SCJ) and distilled water. No other synthetic minerals were added in the media beside sugar cane juice. This is infect the first study in which SCJ is exclusively used for the production of pullulan without adding any other synthetic mineral in the media therefore making it very cost effective.

\section{Hypotheses}

The cost of any polysaccharide mainly depends on the cost of carbon and nitrogen sources used for its production. Due to this the cost of production generally goes high. Therefore it is required to use substances which are cheap and will reduce the cost of production at industrial level. This study was 
mainly on the Hypotheses that by using cheap sources of nutrition we can reduce the cost of production of the polysaccharide in study.

\section{Materials and Methods}

\section{Micro-organisms and growth conditions}

A. pullulans used in this work was isolated from college campus of St. Aloysius' College, Jabalpur, Madhya Pradesh. Isolation was done by selective enrichment method (Singh et al., 2012). The strain was grown on agar medium plates containing Glucose 2.0\%, Ammonium Sulphate $0.06 \%$, di-Potassium Hydrogen Orthophosphate $0.5 \%$, Sodium Chloride $0.1 \%$, Magnesium Sulphate 0.04\% and Yeast Extract $0.04 \%$ with $\mathrm{pH} 5$. Isolates were maintained on the same medium at $4^{\circ} \mathrm{C}$ and sub cultured every fortnight.

\section{Inoculums preparation}

Cell suspension was prepared by inoculating 1 $\mathrm{ml}$ of $48 \mathrm{~h}$ grown culture in $200 \mathrm{ml}$ of the Sugar Cane Juice broth (100mlSugarcane juice and $100 \mathrm{ml}$ distilled water) and incubated at $37^{\circ} \mathrm{C}$ for $24 \mathrm{~h}$ to achieve active exponential phase of the culture.

\section{Effect of time on pullulan production}

The effect of time on pullulan production by A. pullulans using cane juice and distilled water as a medium was studied. The experiments were carried out at a time intervals of $24 \mathrm{~h}$. Pullulan and biomass production was analyzed.

\section{Effect of $\mathrm{pH}$}

In order to investigate the influence of $\mathrm{pH}$ on pullulan production from A. pullulans utilizing cane juice and distilled water, the initial $\mathrm{pH}$ of the medium was adjusted to 2.0, 3.0, 5.0, 7.0, and 9.0, individually, using either $1 \mathrm{~N} \mathrm{HCl}$ or
$1 \mathrm{~N} \mathrm{NaOH}$ and left uncontrolled during the fermentation. Five $\mathrm{ml}$ of the inoculum was used to inoculate $100 \mathrm{ml}$ sterile medium in a $250 \mathrm{ml}$ Erlenmeyer flasks and incubated for $96 \mathrm{~h}$ at $37^{\circ} \mathrm{C}$. The broth was analyzed for pullulan production.

\section{Effect of different temperatures}

The influence of different temperatures on pullulan production from A. pullulans utilizing cane juice and distilled water was investigated. Five $\mathrm{ml}$ of the inoculum was used to inoculate $100 \mathrm{ml}$ sterile medium in a $250 \mathrm{ml}$ Erlenmeyer flasks and incubated for $96 \mathrm{~h}$ at different temperatures viz., $30^{\circ} \mathrm{C}, 37^{\circ} \mathrm{C}$, $43^{\circ} \mathrm{C}, 50^{\circ} \mathrm{C}$ and $60^{\circ} \mathrm{C}$ individually. The fermented broth was analyzed for pullulan production.

\section{Effect of different concentration of cane juice and distilled water}

The influence of different ratio of cane juice and distilled water on pullulan production from $A$. pullulans utilizing cane juice and distilled water was investigated using different concentrations of cane juice in distilled water viz., 20\%, 30\%, 50\%, 70\% and 90\% respectively. Five $\mathrm{ml}$ of the inoculum was used to inoculate $100 \mathrm{ml}$ sterile medium in a $250 \mathrm{ml}$ Erlenmeyer flasks and incubated for $96 \mathrm{~h}$ at $37^{\circ} \mathrm{C}$. The broth was analyzed for pullulan production.

\section{Extraction and estimation of pullulan}

After fermentation, the culture medium was heated at $100^{\circ} \mathrm{C}$ in water bath for 15 minutes cooled to room temperature and centrifuged at $12,000 \mathrm{rpm}$ at $4^{\circ} \mathrm{C}$ for 10 minutes to remove cells and other precipitates. Three milliliters of the supernatant were transferred into a test tube and then $6 \mathrm{ml}$ of the cold ethanol was added to the test tube and mixed thoroughly and held at $4^{\circ} \mathrm{C}$ for $12 \mathrm{~h}$ to precipitate the 
extracellular polysaccharide. After removal of the residual ethanol the precipitate was dissolved in $3 \mathrm{ml}$ of deionized water at $80^{\circ} \mathrm{C}$ and the solution was dialyzed against deionized water for $48 \mathrm{~h}$ to remove small molecules in the solution. The exo polysaccharide was precipitated again by using $6 \mathrm{ml}$ of the cold ethanol and the residual ethanol was removed the precipitate was dried at $80^{\circ} \mathrm{C}$ to a constant weight (Badr-Eldin et al., 1994). Pullulan was measured using electronic balance and expressed in $\mathrm{g} / \mathrm{l}$.

Hydrolysis of the purified extracellular polysaccharide and assay of reducing sugar

To assay the component of the extracellular polysaccharide, the purified precipitate was vacuum desiccated to no alcohol by using a vacuum pump, then dissolved in $3 \mathrm{ml}$ deionized water at $80^{\circ} \mathrm{C}$ in water bath. The dissolved substrate was hydrolyzed by incubating the mixture of $0.5 \mathrm{ml}$ of the substrate, $0.4 \mathrm{ml}$ of $\mathrm{Na}_{2} \mathrm{HPO}_{4}(0.2 \mathrm{M})$, citric acid buffer $0.1 \mathrm{M}(\mathrm{pH} \quad 5.0)$ and $0.1 \mathrm{ml}$ pullulanase (Sigma Chemicals, U.S.A.) for 21 hours at $40^{\circ} \mathrm{C}$. The released reducing sugar was determined by using the modified D.N.S. method (Singh et al., 2012) for the conformation of pullulan.

\section{Statistical analysis}

Karl Pearson Method (Variability) was followed for statistical analysis. All the experiments were done in triplicate and mean were calculated using standard deviation.

\section{Results and Discussion}

Effect of time course on pullulan
production and biomass yield

In order to find an optimum time for pullulan production using cane juice as substrate, the experiments were carried out for different times. The effect of time on the kinetics of pullulan production by $A$. pullulans is shown in Figure 1. The highest concentration of pullulan $(4.9 \pm 0.06 \mathrm{~g} / 100 \mathrm{ml})$ was obtained at a fermentation period of $96 \mathrm{~h}$. Similarly, highest biomass production $(4.2 \pm 0.05 \mathrm{~g} / 100 \mathrm{ml})$ was also obtained at fermentation period of $96 \mathrm{~h}$. The pullulan concentration gradually increases when fermentation time increases and reaches a maximum for a fermentation period of $96 \mathrm{~h}$. After which, the production becomes steady.

\section{Effect of initial $\mathrm{pH}$ on biosynthesis of pullulan}

The effect of $\mathrm{pH}$ (2.0 to 9.0) on the production of pullulan from $A$. pullulans utilizing cane juice and distilled water is shown in Figure 2. Pullulan concentration gradually increased with increasing initial $\mathrm{pH}$ up to 5 and then decreased. The highest pullulan concentration of $4.2 \pm 0.04 \mathrm{~g} / 100 \mathrm{ml}$ was achieved at $\mathrm{pH}$ of 5.0. Beyond this the production decreased.

\section{Effect of temperature on pullulan production}

In order to find an optimum temperature for pullulan production using cane juice and distilled water as substrate, the experiments were carried out for different temperatures.

The effect of temperature on pullulan production by $A$. pullulans is shown in Figure 3. It is clearly indicated in Figure 3 that strain has able to produce high amount of pullulan $(4.3 \pm 0.03 \mathrm{~g} / 100 \mathrm{ml})$ at $37^{\circ} \mathrm{C}$. Beyond this the production decreased significantly.

\section{Effect of different concentration of cane} juice and distilled water

Carbon sources play a vital role in the production of pullulan (Singh et al., 2012; Singh et al., 2016; Singh et al., 2017) (Fig. 4). 
Fig.1 Effect of fermentation time on biosynthesis of pullulan production

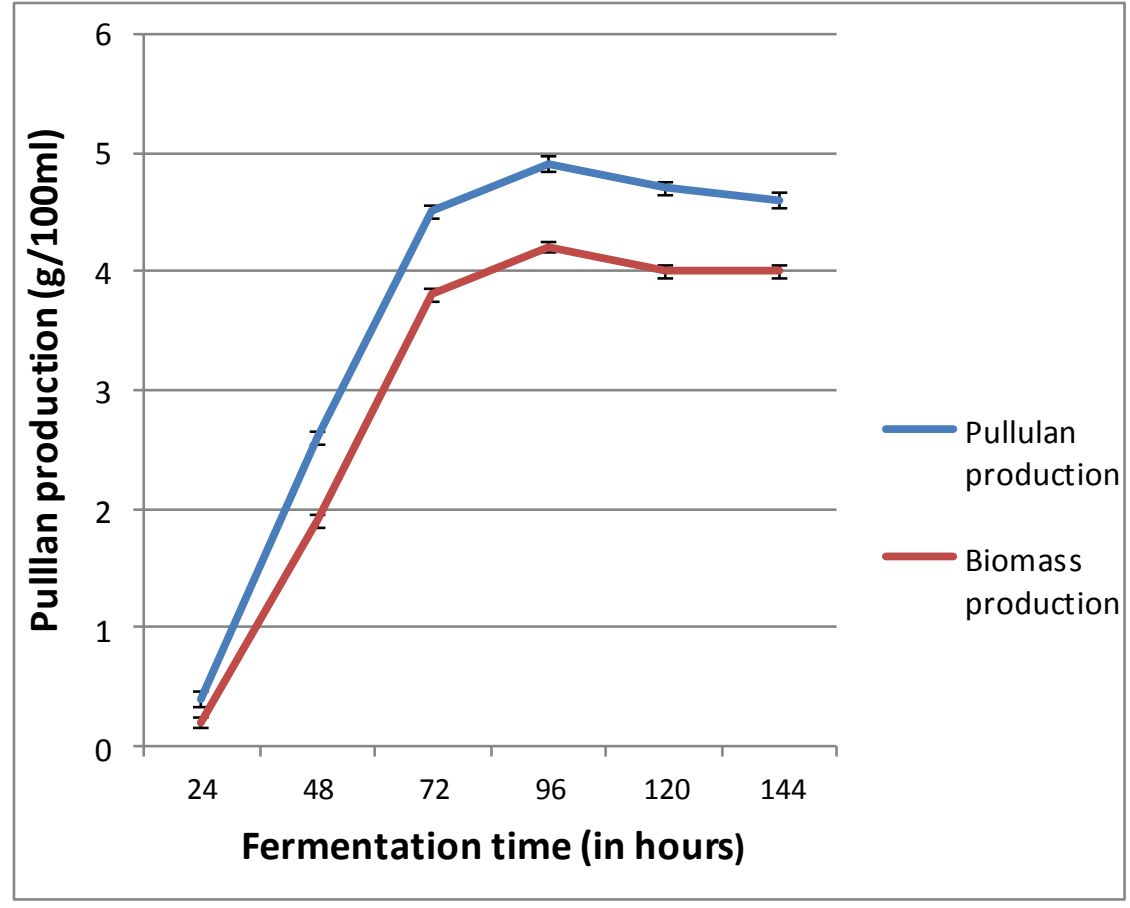

Fig.2 Effect of initial $\mathrm{pH}$ on biosynthesis of pullulan

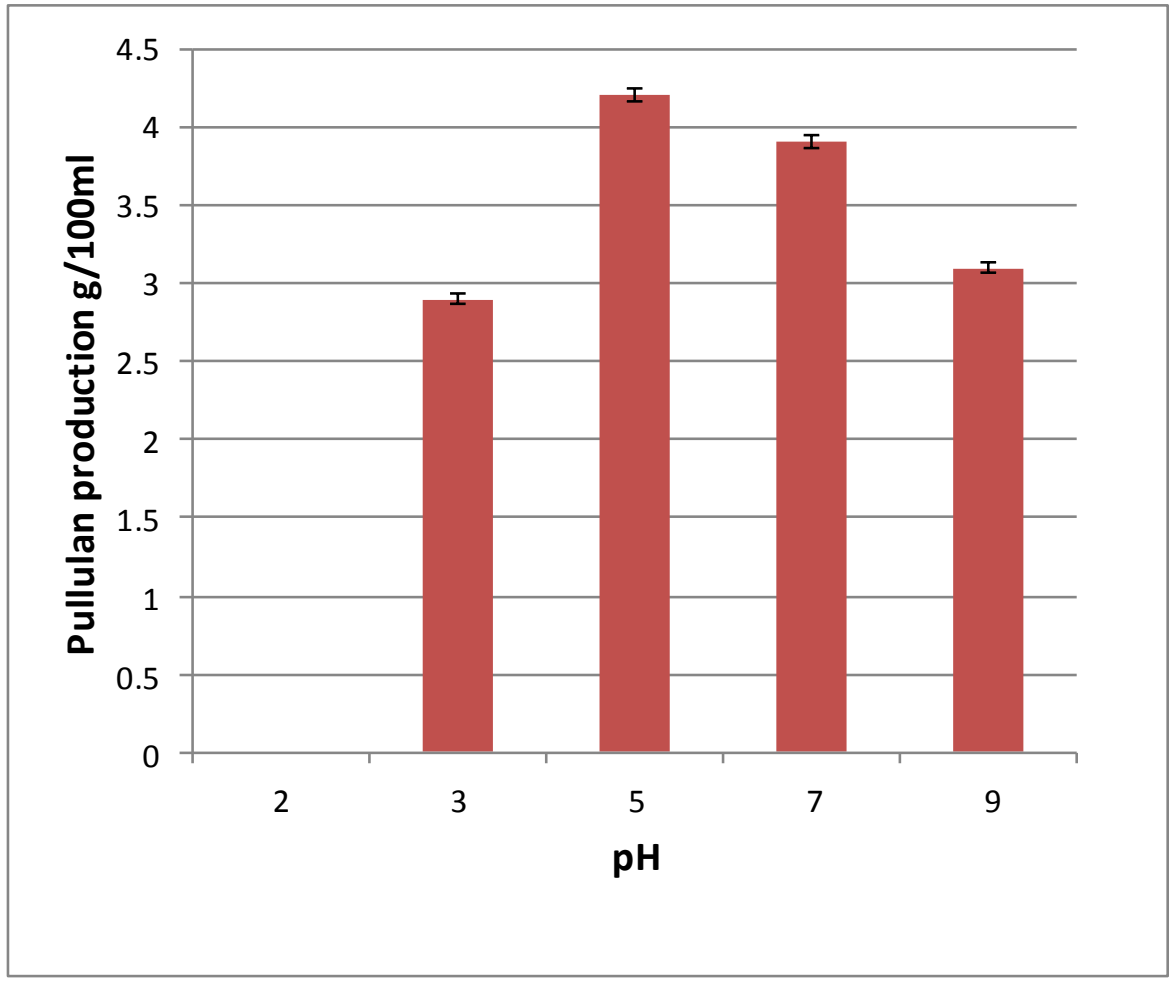


Fig.3 Effect of fermentation time on biosynthesis of pullulan production

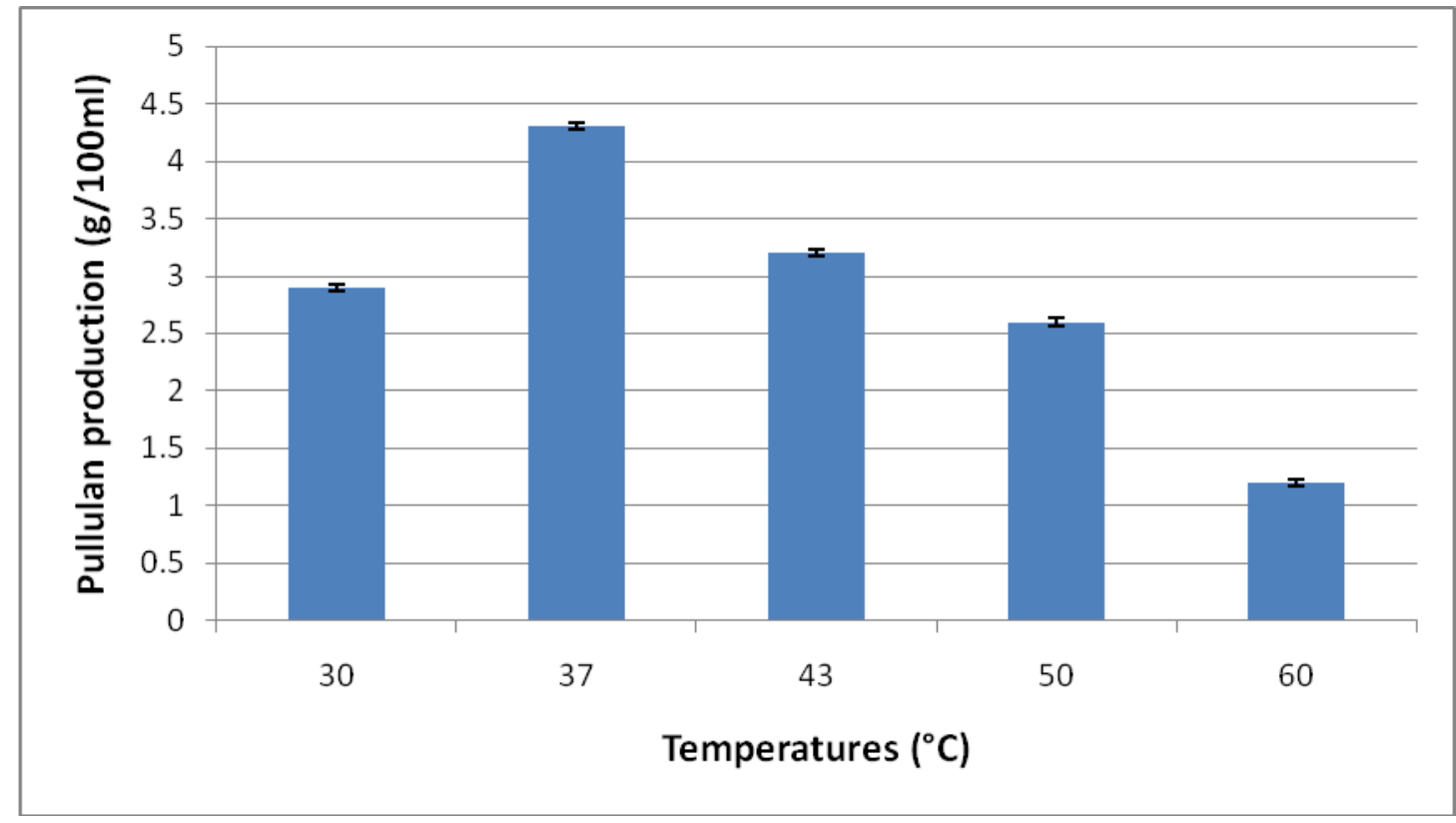

Fig.4 Effect of different concentration of cane juice and water

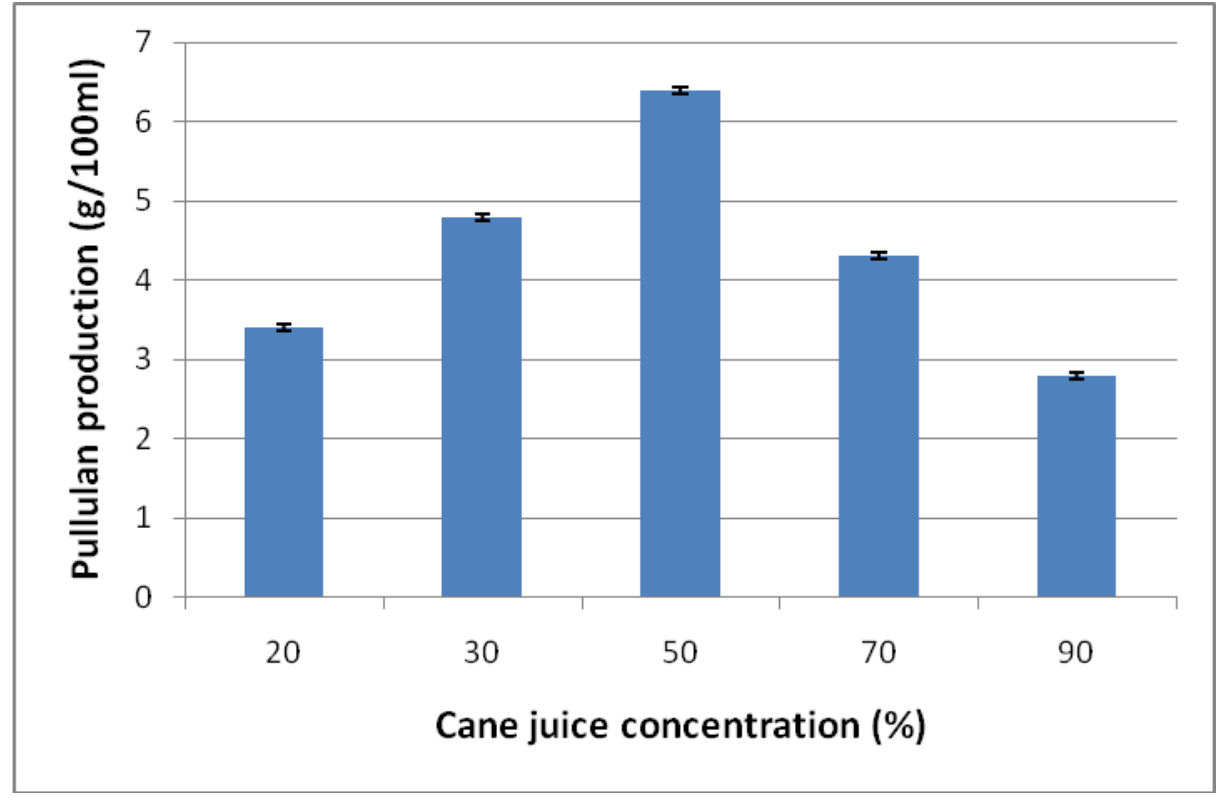

The influence of different ratio of cane juice and distilled water on pullulan production from A. pullulans was investigated. Among different ratio of cane juice and distilled water, $50 \%$ cane juice concentration showed best result $(6.4 \pm 0.04 \mathrm{~g} / 100 \mathrm{ml})$. Beyond this ratio pullulan production dropped significantly. Pullulan production is directly related to yeast phase of growth. Yeast-like cells are mainly responsible for pullulan production (Cambell et al., 2004). Incubation period for pullulan production varies from strain to strain; therefore incubation period has been evaluated for pullulan and biomass 
production. From the study it was revealed that highest production of pullulan was obtained at $96 \mathrm{~h}$ of fermentation. Further after 96h of incubation the production of pulluan became stable. This was mainly because the fungus did not produce pullulan degrading enzyme pullulanase.

Similar trend in pullulan production was observed by in other works of same worker (Singh et al., 2012; Singh et al., 2016; Singh et al., 2017). Similarly maximum biomas production was also observed at 96h, since formation of biomass directly depends on pullulan formation. Maximum pullulan production was achieved when the cells reached their stationary phase which was at 96h and beyond this no further growth in the cells were seen, thus production of pullulan became stable.

It has been reported that $\mathrm{pH}$ has profound effect on both the rate of production and synthesis of pullulan. Different workers have reported pullulan production at different $\mathrm{pH}$ range (Singh et al., 2012; Singh et al., 2016; Singh et al., 2017; Thirumavalan et al., 2008; Thirumavalan et al., 2009).

In this particular study maximum production of pullulan was obtained at a $\mathrm{pH}$ 5. Optimal $\mathrm{pH}$ values for pullulan production depends on different yeast strain, composition of the fermentation medium and growth conditions. Therefore, the physiological function of $A$. pullulans varies from strain to strain in case of $\mathrm{pH}$ also. This is perhaps due to either special structure of the membrane and cell wall or transport system of the organism along with the change of cytosol $\mathrm{pH}$ due to medium constituents affecting the critical level at specific pka value of the medium and ultimately affecting more or less hydrogen ion concentration which in turn affected cell growth or pullulan synthesis. At very low $\mathrm{pH}$ no growth was seen due to acid production in the medium by the yeast cells which negatively affects the growth of fungus and production of polysaccharide.

Fermentation temperature is one of the most important factors for pullulan production affecting yeast phase of $A$. pullulans growth because change in morphology adversely affects pullulan production. In A. pullulans yeast form is mainly responsible for pullulan production (Campbell et al., 2004). In this study highest pullulan production was seen at $37^{\circ} \mathrm{C}$. This means that this particular strain is thermo-tolerant which can tolerate high temperature. Generally fungus grows at a range of $28-32^{\circ} \mathrm{C}$ but our strain can grow at much higher level than that. This is an important finding because in industry temperature goes up very high and only thermo-tolerant strains are able to tolerant it thus making it a suitable strain for industrial use.

Sugarcane juice along with distilled water was used for the production of pullulan. It was seen that maximum production was seen at $50 \%$ of sugarcane juice concentration. Lesser concentration did not support the growth of fungus due to low concentration of sugar and minerals. Higher concentration also did not support the growth due to more concentration of sugar and minerals which caused a nutrient shock to the fungus. Thus the best result was seen at 50\% concentration. This is the first study done in which no synthetic mineral is used for the production of pullulan. Polysaccharide is grown on a media consisting of Sugar Cane Juice (SCJ) and distilled water thus making it very cheap and cost effective.

From the ongoing study it can be concluded the isolate of Aureobasidium pullulans was able to produce higher amount of pullulan $(6.4 \pm 0.04 \mathrm{~g} / 100 \mathrm{ml})$ in a medium composed of Sugarcane juice and distilled water. This is 
the first study done in which no synthetic mineral is used for the production of pullulan. Polysaccharide is grown on a media consisting of Sugar Cane Juice (SCJ) and distilled water thus making it very cheap and cost effective.

\section{Acknowledgement}

The corresponding author (Ranjan Singh) is thankful to the Head, Department of Botany and Microbiology, St. Aloysius College (Autonomous), Jabalpur, M.P., India for the necessary laboratory facility provided to carry out the research. A thank is also extended to the Principal, St. Aloysius College (Autonomous), Jabalpur, M.P., India for providing the necessary chemicals and glassware required to carry out the research work.

\section{Conflict of Interest}

There is no conflict of interest among the authors related to the work.

\section{References}

Badr-Eldin, S.M., El-Tayeb, O.M., El-Masry, H.G., Mohamad, F.H.A., and Abd ElRahman, O.A. (1994). Polysacharide production by Aureobasidium pullulans: Factors affecting polysaccharide formation. World Journal of Microbiology and Biotechnology, 10, 423-426.

Barnett, C., Smith, A., Scanlon, B., and Israilides, C. J. (1999). Pullulan production by Aureobasidium pullulans growing on hydrolysed potato starch waste. Carbohydrate Polymers, 38, 203-209.

Boa, J. M., and LeDuy, A. (1984). Peat hydrolysate medium optimization for pullulan production. Applied Environmental Microbiology, 48, 26-30.
Boa, J. M., and LeDuy, A. (1987). Pullulan from peat hydrolysate fermentation kinetics. Biotechnology Bioengineering, 30, 463-470.

Campbell, B. S., Siddique, A. B. M., McDougall, B. M., and Seviour, R. J. (2004). Which morphological forms of the fungus Aureobasidium pullulans are responsible for pullulan production? FEMS Microbiology Letters, 232, 225228.

Chao, A., Ma, S. J., Chang, F., and Xue, W. J. (2017). Efficient production of pullulan by Aeruobasidium pullulans grown on mixture of potato starch hydrolysate and sucrose. Brazilian Journal of Microbiology, 48, 180-185.

Gaur, R., Singh, R., Gupta, M., and Gaur, M. K. (2010). Aureobasidium pullulans, an economically important polymorphic yeast with special reference to pullulan. African Journal of Biotechnology, 9 (47), 7989-7997.

Israilides, C. J., Smith, A., Harthill, J. E., Barnett, C., Bambalov, G., and Scanlon, B. (1998). Pullulan content of the ethanol precipitate from fermented agro-industrial wastes. Applied Microbiology and Biotechnology, 49, 613-617.

Leather, T. D., and Gupta, S. C. (1994). Production of pullulan from fuel ethanol byproducts by Aureobasidium sp. strain NRRY-12,974. Biotechnology Letters, 16, 1163-1166.

Roukas, T. (1998). Pretreatment of beat molasses to increase pullulan production. Process Biochemistry, 33, 805-810.

Roukas, T. (1999). Pullulan production from brewery wastes by Aureobasidium pullulans. World Journal of Microbiology and Niotechnology, 15, 447-450.

Roukas, T., and Biliaderis, C. G. (1995). Evaluation of carob pod as a substrate 
for pullulan production by Aureobasidium pullulans. Applied Biochemistry and Biotechnology, 55, 27-44.

Roukas, T., and Liakopoulou, K. M. (1999). Production of pullulan from beet molasses by Aureobasidium pullulans in a stirred tank fermentor. Journal of Food Engineering 40, 89-94.

Singh, R., Gaur, R., Bansal, S., Biswas, P., Pandey, P. K., Jamal, F., Tiwari S, and Gaur, M.K. (2015). Aureobasidium pullulans- an industrially important pullulan producing black yeast. International Journal of Current Microbiology and Applied Sciences, 4(10), 605-622.

Singh, R., Gaur, R., Bansal, S., Biswas, P., Pandey, P. K., Jamal, F., Tiwari S, and Gaur, M.K. (2016). Production of pullulan from a high yielding strain of Aureobasidium pullulans isolated from Jabalpur region of Madhya Pradesh in Central India. Journal of Chemical and Pharmaceutical Research, 8(8), 126132.

Singh, R., Gaur, R., Bansal, S., Jamal, F., Pandey, P. K., Tiwari, S, Sarsaiya, S., Mishra, S., Chaturvedi, N., Singh, D. P., Gaur, M. K. and Arasu, G.V. (2017). Production of pullulan from a high yielding strain of Aureobasidium pullulans in non-stirred flask type fermentation system. Journal of Microbiology and Biotechnology Research, 7(1), 26-32.
Singh, R., Gaur, R., Tiwari, S., and Gaur, M. K. (2012). Production of pullulan by a thermotolerant Aureobasidium pullulans strain in non-stirred fed batch fermentation process. Brazilian Journal of Microbiology, 43, 1042-1050.

Thirumavalan, K., Manikkandan, T. R., and Dhanesekar, R. (2008). Batch fermentation kinetics of pullulan from Aureobasidium pullulans using low cost substrates. Biotechnology, 7, 317-322.

Thirumavalan, K., Manikkandan, T. R., and Dhanesekar, R. (2009). Pullulan production from coconut by-products by Aureobasidium pullulans. African Journal of Biotechnology, 8(2), 254258.

Vijayendra, S. V. N., Bansal, D., Prasad, M. S., and Nand, K. (2001). Jaggery: A novel substrate for pullulan production by Aureobasidium pullulans CFR-77. Process Biochemistry, 37, 359-364.

Xia, J., Xu, J., Liu, X., Xu, J., Wang, X., and Li, X. (2017). Economic co-production of poly (malic acid) and pullulan from Jerusaleum artichoke tuber by Aureobasidium pullulans HA-4D. Bmc Biotechnology, 17 (20), 1-10.

Youseef, F., Biliaders, C. G., and Roukas, T. (1984). Enhancement of pullulan production by Aureobasidium pullulans in batch culture using olive oil and sucrose as carbon sources. Applied Biochemistry Biotechnology. 74, 13-30.

\section{How to cite this article:}

Ranjan Singh, Rajeeva Gaur, Prabhash K. Pandey, Farrukh Jamal, Laxmi K. Pandey, Harish K. Kewat, Soni Tiwari, Pritha Biswas and Manogya K. Gaur. 2018. A Novel Media Optimized for Production of Pullulan in Flask Type Fermentation System. Int.J.Curr.Microbiol.App.Sci. 7(04): 53-61. doi: https://doi.org/10.20546/ijcmas.2018.704.007 